\title{
Dental Implant Placement by Using Metal Reinforced Acrylic Stent - An Innovative Technique
}

\author{
MC Prasant ${ }^{1}$, Ziyauddin ${ }^{2}$, Rohit Kumar Singh², Tushanshu Soni², Shiv Pratap Singh ${ }^{2}$, Shreya Chouksey ${ }^{3}$ \\ ${ }^{1}$ Professor and HOD, Department of Oral and Maxillofacial Surgery, RKDF Dental College, Bhopal, Madhya \\ Pradesh, India
}

${ }^{2}$ PG Resident, Department of Oral and Maxillofacial Surgery, RKDF Dental College, Bhopal, Madhya Pradesh, India

${ }^{3}$ PG Resident, Department of Prosthodontics and Crown and Bridge), Hitkarni Dental College and Hospital, Jabalpur, Madhya Pradesh, India

*Corresponding Author: Ziyauddin, PG Resident, Department of Oral and Maxillofacial Surgery, RKDF Dental College, Bhopal, Madhya Pradesh, India; Email: drkhanziya9494@gmail.com

Received Date: 28-01-2022; Accepted Date: 17-02-2022; Published Date: 24-02-2022

Copyright $^{\odot} 2022$ by Ziyauddin, et al. All rights reserved. This is an open access article distributed under the terms of the Creative Commons Attribution License, which permits unrestricted use, distribution and reproduction in any medium, provided the original author and source are credited.

\begin{abstract}
Since the Branemark method, implant surgery has progressed significantly as well as its delayed impact on the edentulous jaw. 2 A surgical guide, also known as a stent, is essential for implant placement at correct location and angulation. Transferring productive and prosthetic arrangement details to the surgical stage is evaluative.
\end{abstract}

\section{Keywords}

Flap Indication; Implant Placing; Implant Surgical Pilot

\section{Introduction}

The surgical guide is critical in allowing for proper implant location and angulation. The transfer of diagnostic and prosthetic planning information to the surgical phase is critical. Ideal 
Prerequisites- It should be rigid buccolingually, mesio distally and apico coronally in the correct position [1-3].

The surgical guides' extensions, if there are any residual teeth in the arch being treated, the template should be able to fit over and/or around them to support and position the guiding stent. The template might be enlarge onto thoughtless soft tissue regions, such as the palate and tuberosities in the maxilla or the retromolar pads in the mandible, when no residual teeth are present. The guidance template can then be employed once the soft tissue has been reflected from the implant location and during the implant osteotomy preparation. Depending on the operation area Sites that are partially edentulous have their own set of guidelines. Toothsupported surgical guides can be used in short-span partially edentulous arches and tooth- and bone-supported implant surgical guides can be used in long-span partially edentulous arches. Guides for a completely edentulous site include the following: Supported by mucosa or bone based on the backing: Teeth, Bone and Mucosa provide support for the surgical guides $[3,4]$. The use of various types of surgical guides is governed by a set of rules. Guides with tooth support: have to be least three stable teeth to help this pilot in surgery [3].

- Mucosa-supported guides - These are employed in edentulous sites. There is little or no tissue reflection required, resulting in minimal postoperative discomfort. During surgery, a scan prosthesis and surgical guidance will be required [3].

- Bone-supported guides - These are used in both partially and totally edentulous sites. It should have minimum of $3 \mathrm{~cm}$ of bone upon which it lies when used incomplete edentulous sites, or three teeth will need to be replaced. Bone guides are particularly useful in edentulous areas when the bone is thin. The raised flap should provide for a clear view of the implant locations and the placement of guides [3].

A well-developed plan should be correctly conveyed, leaving less room for changes during operation.

After completion of the osteotomy and flattening of the bone, bone-based drill guides in sequence were placed over the newly flattened bone to execute osteotomies accurately with sequenced drill protocols for implantation, each to carefully expand the osteotomy to manufacturer's directed diameters to ensure proper placement of the dental implant [5].

To accomplish correct implant placement within the bone, the templates regulated depth, trajectory and diameter [2]. 
- Reduces the number of manual errors associated with implant implantation by hand. Because surgical guides allow for little involvement, postoperative surgical issues are minimised, benefiting both the patient and the practitioner psychologically [1].

Precision- Implants are prosthetically driven components and any variation can result in unexpected functional outcomes. Implant placement has grown more precise thanks to surgical guidance. When inserting implants in vital parts of the mouth, the most important consideration is safety. Even the tiniest mistake can result in serious consequences. Such aberrations can be avoided with the use of guidance. Damage to vital structures can be readily avoided.

Predictability- It is impossible to retain predictability and alertness throughout the entire procedure. In comparison to the guided insertion procedure, even expert hands are associated with lower quality.

Aesthetic- The use of surgical guidance to transition from software planning to real implantation has been found to yield satisfactory cosmetic results [3].

Hygiene- Correct implant placement ensures oral hygiene and proper oral health. Implantsupported prostheses should be positioned in predetermined positions for the best chance of survival. Such excellent implant placements can be aided by guides [Fig. 1-5] [6].

- Pilot guides - Only pilot drilling are permitted with the sleeves. Control of angulation has been obtained. Manual depth control is obtained by examining marks on drills. The osteotomy site is extended after removal of surgical pilot drill

- Drill guides - Utilizes drill keys to provide complete drill guidance. Different sleeves are used for different drill sizes, which are replaced at the same time as the osteotomy is expanded. The guide controls the angulation as well as the size of the osteotomy; the depth is manually regulated [3]

- Safe/easy guides - Follow the same drill keys as above, but with an extra implant stopper to restrict drilling depth. Allows for the preparation of osteotomies using surgical drills and also in the implant placement [6]

\section{Technique}

1. Make a diagnosis and make a treatment plan $[3,7]$

2. Utilizing facebowtransfer, mount the diagnostic casts made with Type III dental stone (Die) on a semiadjustable articulator 
3. Apply baseplate wax to the undercut areas around the teeth close to the edentulous area and apply cold mould seal/other separating medium to the area

4. Apply autopolymerizing clear acrylic resin to the waxed-up teeth and teeth next to the edentulous area, extending up to the sulcus depth on the lingual side and the occlusal third of the teeth on the buccal side while maintaining the thickness at $1.5 \mathrm{~mm}$

5. Extend the guidance beyond the operation site to the edentulous area if one of the adjacent teeth is gone

6. After the resin has polymerized, indicate a location on the surgical guide that corresponds to the central fossa of the waxed up teeth

7. Remove the guide and waxed-up teeth, leaving a hollow hole in the mouth

8. Place the guide again on the site and apply cold mould seal to the non-tooth bearing area

9. Cover the edentulous area with autopolymerizing clear acrylic resin, extending buccally over the ridge's slope till the depth of sulcus. Maintain a $1.5 \mathrm{~mm}$ flange thickness

10. Place the cast on a milling machine or a conveyor

11. Using the parallel long axis of the adjacent teeth, determine the best implant placement orientation and mark a line upon the cast to show it

12. Attach a no. 2 parallel cutter, crosscut, round tip machine to the guide and drill a hole at the designated location. Bring the handpiece down to the point where another hole is drilled on the flange over the edentulous area, following the chosen implant placement direction

13. On the occlusal side of the guide, cut a groove that extends up to the hole and is open on the buccal side

14. Remove the guide from the cast for trimming, finishing and polishing

15. Usage of the guide-The guide should be retained in position after the flap has been reflected so that the buccal flange should go under the flap, preventing the same from returning to the site of osteotomy

16. Drill sequence

17. Insert the tip of the $2 \mathrm{~mm}$ osteotomy drill into the hole created on the edentulous area to create two points of contact, one at occlusal and another at ridge level and complete the osteotomy

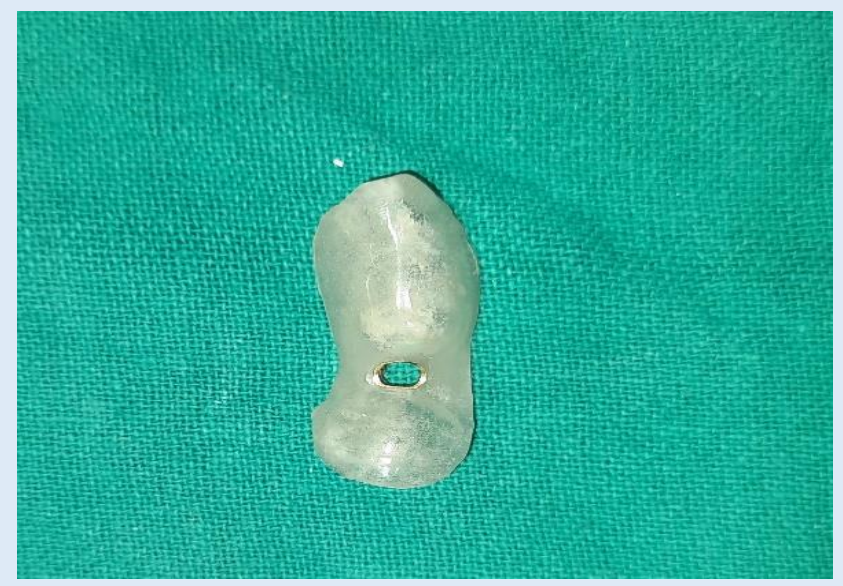

Figure 1: Surgical quide-axial view.

MC Prasant | Volume 3; Issue 1 (2022) | JDHOR-3(1)-042 | Research Article

Citation: Ziyauddin, et al. Dental Implant Placement by Using Metal Reinforced Acrylic Stent - An Innovative Technique. J Dental Health Oral Res. 2022;3(1):1-9. 


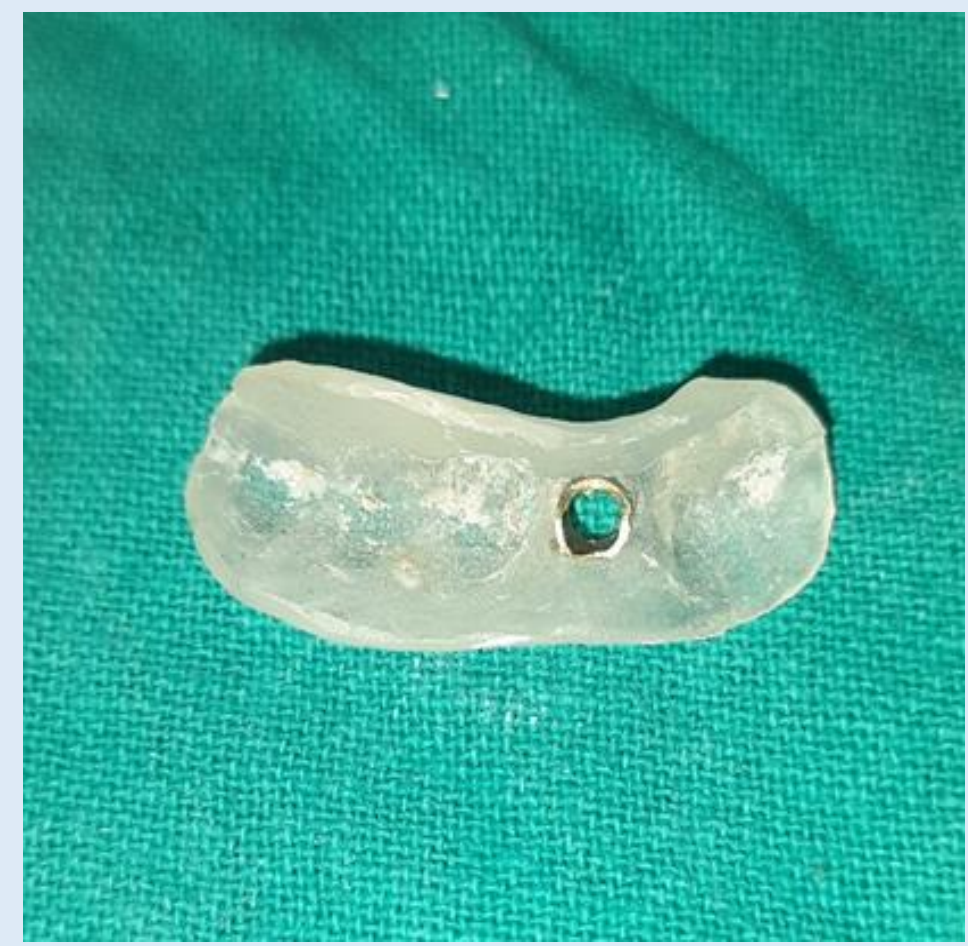

Figure 2: Surgical quide-buccal view.

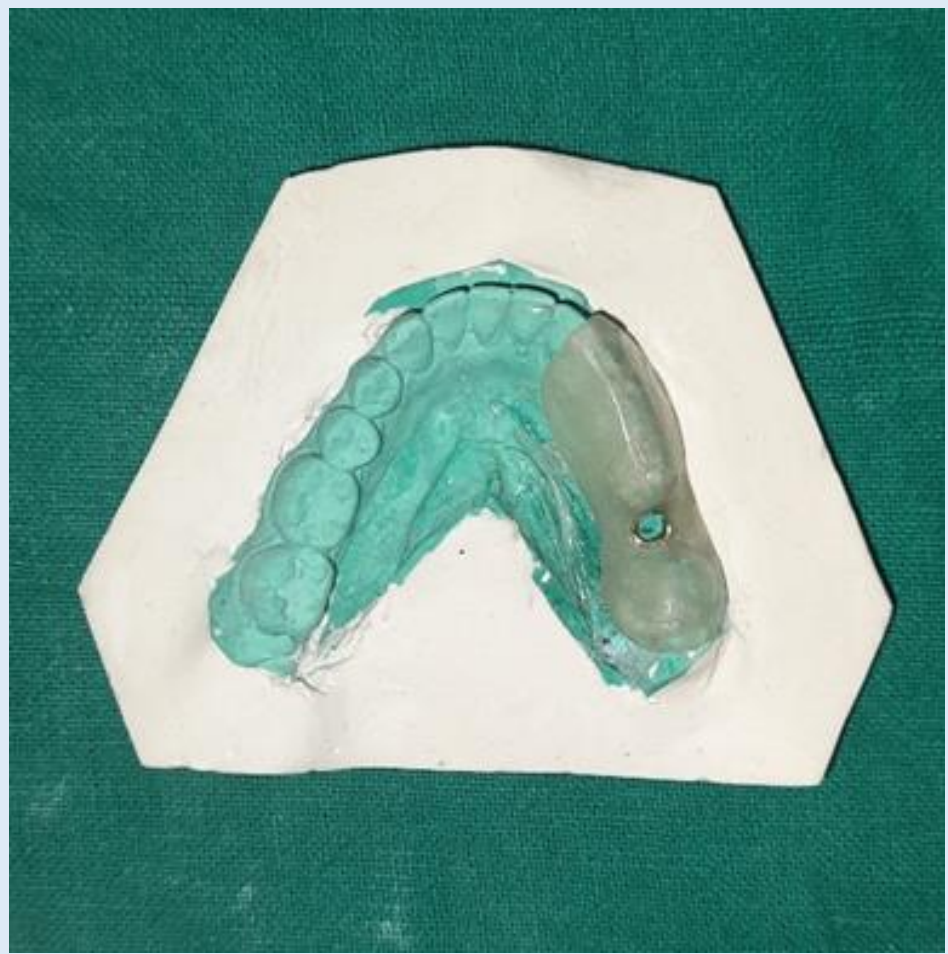

Figure 3: Surgical quide in position on the cast.

MC Prasant | Volume 3; Issue 1 (2022) | JDHOR-3(1)-042 | Research Article

Citation: Ziyauddin, et al. Dental Implant Placement by Using Metal Reinforced Acrylic Stent - An Innovative Technique. J Dental Health Oral Res. 2022;3(1):1-9. 


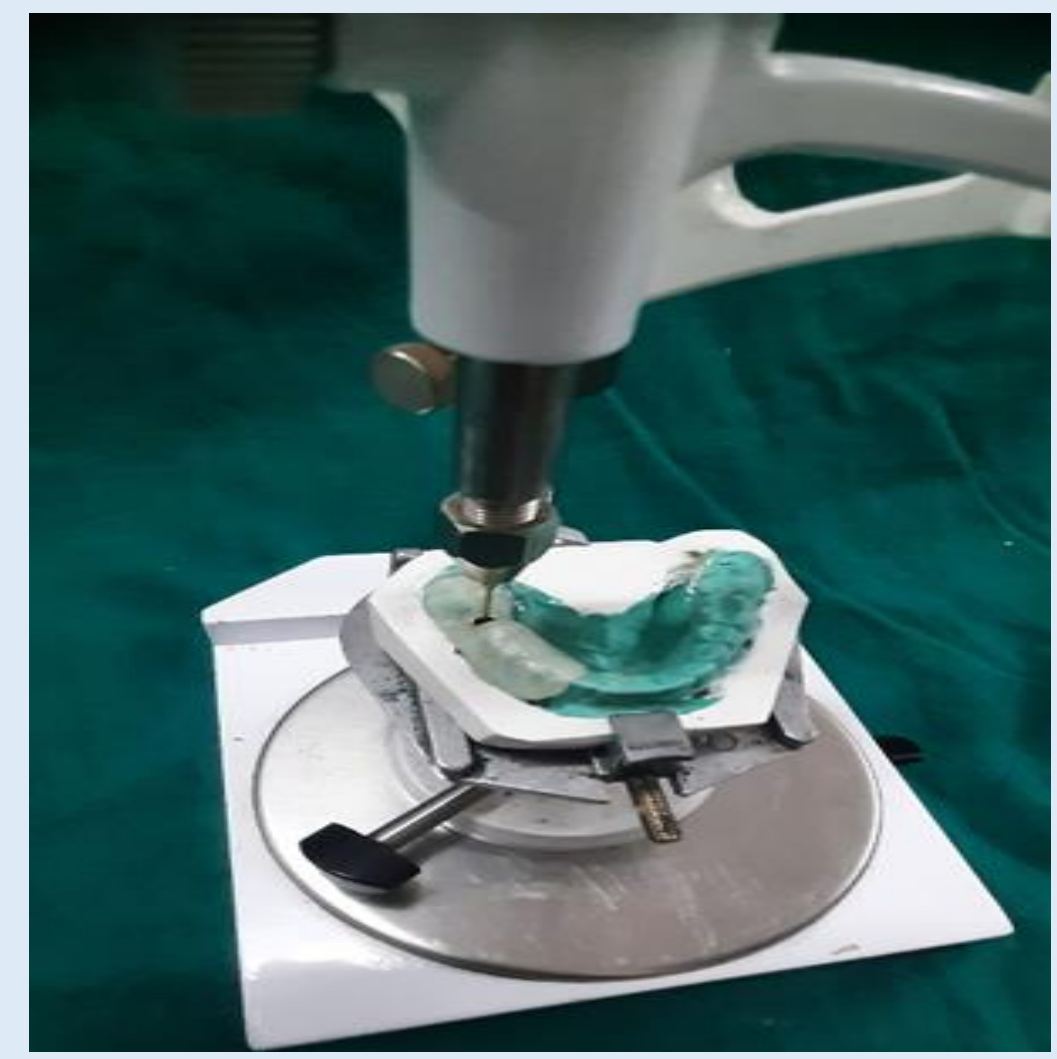

Figure 4: Dental surveryor mandrill check the direction of implant placement.

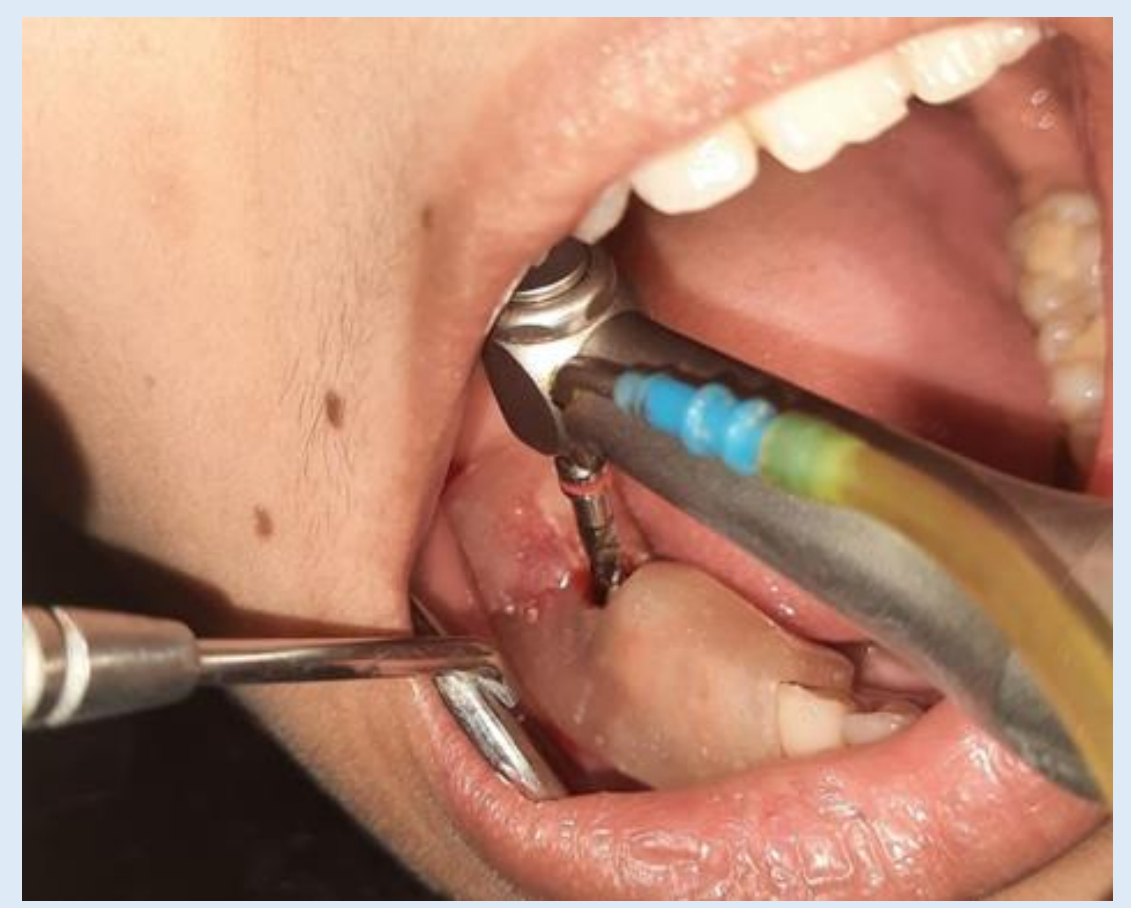

Figure 5: Flap kept away by the surgical guide and drill in position.

MC Prasant | Volume 3; Issue 1 (2022) | JDHOR-3(1)-042 | Research Article

Citation: Ziyauddin, et al. Dental Implant Placement by Using Metal Reinforced Acrylic Stent - An Innovative Technique. J Dental Health Oral Res. 2022;3(1):1-9. 


\section{Discussion}

The current procedure uses a streamlined approach to meet both parameters for optimal implant placement and can be completed in the dentist office by the practitioner. During osteotomy preparation, the surgical guide must assist in transferring the predefined angulation and controlling the location of the drill in all three dimensions. For this it requires at least two reference points: one at the level of crest and another at the occlusal level, roughly $8 \mathrm{~mm}$ [1]. A line can be drawn between these two points to illustrate the pathway of an ideal implant placement. The ideal angulation is perpendicular to the occlusal plane and collateral to the most anterior abutment (natural or implant) conneced to the implant [1]. A $2 \mathrm{~mm}$ twist drill is used to determine the direction and depth of the osteotomy. Following this specified initial drill, more osteotomies will be performed. In the proposed surgical guide, the two points are located as a small opening on the occlusal aspect and a hole on the flange above the crest of the ridge [1].

The procedure described is intended to help clinicians prepare precise osteotomy sites with greater visibility and accessibility. The guide's design includes a window that opens toward the buccal side, which increases visibility. The flap has a flange that extends onto the slope of the ridge, keeping it away from the osteotomy site and preventing it from falling back onto the surgical site. With this an assistant also visualizes the surgical site without interrupting the surgeon to guide for correct positioning and depth [2]. A small aperture is given on the occlusal side so that the pilot drill could be placed in accurate position from the buccal side rather than the occlusal side in cases when the patient has reduced mouth opening or when the implant has to be placed most posteriorly. Two factors influence the optimal prosthetic outcome of an implant prosthesis [1]. Position of the transosteal region of implant body and Direction of the implant body. The direction of the implant body determines whether an angulated abutment or customised abutment is required. Supplementary elements like these will increase the procedure's cost. The easiest way to get the exact location for an implant that meets both parameters is to use entirely limiting surgical guides. However, for a wider community in developing nations, surgical guides made from CAD-CAM as entirely limiting guides are still not economically practical.

The current procedure uses a streamlined approach to meet both parameters for optimal implant placement and can be completed in the dentist office by the practitioner. The above-mentioned new approach to constructing the surgical guide enables for exact implantation in the desired position, resulting in a expected outcome of treatment [Fig. 6]. This surgical guide is also used in flapless surgery in circumstances when enough alveolar bone width available. 
Due to anatomical constraints and improved prosthetics, the surgeon must obtain greater precision in the surgical placement of dental implants [3,7]. During oral implant insertion, the surgeon must guide the drill (angulation, depth and position) according to the ultimate form of the prosthesis. The development of favourable forces on the implants and prosthesis is made easier with ideal placement [6].

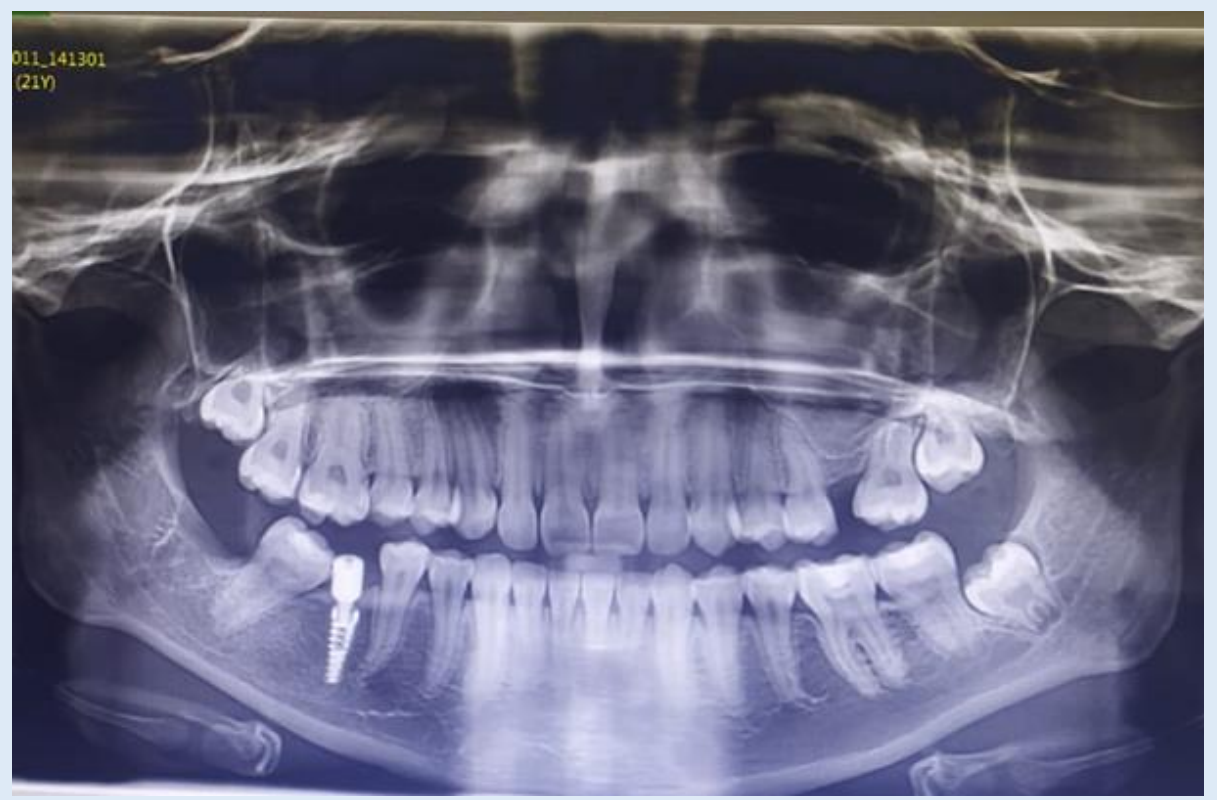

Figure 6: Immediate post-op OPG.

\section{Conclusion}

Surgical guide's help clinicians create good implant prosthetics that are aesthetically pleasing, functional and easy to maintain. The creation of a surgical guide for perfect implant placement by allowing an operator appropriate visibility and accessibility is described in this article. This is a simplified surgical guide fabrication process that may be done using common materials and can be used in a variety of clinical scenarios.

\section{Conflict of Interest}

There are no conflicts of interest.

\section{References}

1. Handelsman M. Surgical guidelines for dental implant placement. Br Dent J. 2006;201(3):139-52.

MC Prasant | Volume 3; Issue 1 (2022) | JDHOR-3(1)-042 | Research Article

Citation: Ziyauddin, et al. Dental Implant Placement by Using Metal Reinforced Acrylic Stent - An Innovative Technique. J Dental Health Oral Res. 2022;3(1):1-9. 
2. Mijiritsky E, Ben Zaken H, Shacham M, Cinar IC, Tore C, Nagy K, et al. Variety of surgical guides and protocols for bone reduction prior to implant placement: a narrative review. Int J Environ Res Public Health. 2021;18(5):2341.

3. Khanna SS, Munde BS, Baisane PM, Shujaulla S, Tabasum ST, Shammas M. Surgical guides in implants: A Review. 2020.

4. Anunmana C, Ueawitthayasuporn C, Kiattavorncharoen S, Thanasrisuebwong P. In vitro comparison of surgical implant placement accuracy using guides fabricated by three different additive technologies. Applied Sci. 2020;10(21):7791.

5. Reddy VA, Reddy V, Reddy Gade CK. Guided surgery for a simple and predictable implant placement. J Interdisciplinary Dent. 2012;2(2).

6. Umapathy T, Jayam C, Anila BS, Ashwini CP. Overview of surgical guides for implant therapy. J Dental Implants. 2015;5(1):48.

7. Pawar A, Mittal S, Singh RP, Bakshi R, Sehgal V. A step toward precision: a review on surgical guide templates for dental implants. Int J Sci Study. 2015;2(11):262-6. 